\title{
Atrial natriuretic peptide: an essential physiological regulator of transvascular fluid, protein transport, and plasma volume
}

\author{
Fitz-Roy E. Curry \\ Department of Physiology and Membrane Biology, School of Medicine, University of California Davis, Davis, California, USA.
}

\begin{abstract}
Atrial natriuretic peptide (ANP) acts acutely to reduce plasma volume by at least 3 mechanisms: increased renal excretion of salt and water, vasodilation, and increased vascular permeability. Authors of a study in this issue of the JCI performed a knockout of the receptor for ANP in vascular endothelia in order to distinguish the effects of ANP-dependent increases in vascular permeability from those of other endocrine actions of ANP in the regulation of plasma volume (see the related article beginning on page 1666). The knockout mice exhibited reduced vascular permeability to plasma protein, resulting in chronically increased plasma volume, arterial hypertension, and cardiac hypertrophy. Renal excretion and vasodilation did not account for these changes. Thus ANP-induced increases in endothelial permeability may be critical to the ability of ANP to lower arterial blood pressure.
\end{abstract}

\section{Renal versus endothelial mechanisms in regulating plasma volume}

Plasma volume is maintained by the interplay of mechanisms regulating total extracellular fluid volume and Starling forces, the latter of which govern the distribution of the extracellular fluid volume between the vascular space and the interstitial space (1). For example, atrial natriuretic peptide (ANP) is a small peptide secreted by the heart upon atrial stretch and high systemic blood pressure. The acute effects of this potent, shortlived peptide include increased glomerular filtration and increased renal excretion of sodium and water. These changes may serve to decrease blood volume and subsequently lower blood pressure $(2,3)$. However, if these renal responses were the primary effects of ANP action, the loss of tubular fluid derived from glomerular filtration of plasma would concentrate the plasma proteins in the vascular space. The resultant increase in the osmotic pressure of the plasma protein concentration would favor reabsorption of fluid across the endothelial barrier from the interstitial space. Thus, the tendency of ANPdependent renal mechanisms to decrease

Nonstandard abbreviations used: ANP, atrial natriuretic peptide; BNP, B-type natriuretic peptide; GC-A, guanylyl cyclase-A.

Conflict of interest: The author has declared that no conflict of interest exists.

Citation for this article: J. Clin. Invest. 115:1458-1461 (2005). doi:10.1172/JCI25417. plasma volume would be opposed by the change in Starling forces, which would tend to increase plasma volume.

In addition to these renal effects, ANP causes both vasodilation, by relaxing vascular smooth muscle, and an acute increase in vascular permeability via receptors on the microvascular endothelium $(4,5)$. These mechanisms also decrease blood volume by favoring redistribution of plasma protein and fluid from the vascular space to the interstitial space. Thus, a key question concerning the role of ANP in the regulation of plasma volume is the relative importance of the renal versus the nonrenal actions of ANP. Renkin and Tucker (5) argued that the extrarenal actions of ANP enable it to preferentially regulate plasma volume. The study reported by Sabrane, Kuhn, and colleagues in this issue of the JCI (6) provides new data to support this hypothesis by investigating the regulation of plasma volume in transgenic mice in which one of the common receptors for ANP has been selectively deleted in vascular endothelium.

\section{Selective deletion of the endothelial receptor for ANP}

Over the past decade, investigators have examined a number of mouse models in which global deletion of the genes for ANP, the closely related B-type natriuretic peptide (BNP), or the ANP/BNP receptor, guanylyl cyclase-A (GC-A; also known as natriuretic peptide receptor 1 ), resulted in varying degrees of hypertension, expanded plasma volumes, and cardiac hypertrophy. These results support the hypothesis that the common ANP/BNP receptor, GC-A, signaling via a guanylyl cyclase pathway, is essential for plasma volume regulation but do not enable evaluation of the relative contributions of renal, vascular smooth muscle, and endothelial cell-mediated mechanisms to this regulation. An important step toward the goal to evaluate the contribution of each mechanism was made when Holtwick, Kuhn, and colleagues used Crelox technology to selectively delete GC-A in the vascular smooth muscle cells of mice (7). The mutant mice were not hypertensive, suggesting that the direct vasodilating actions of ANP on vascular smooth muscle cells do not contribute significantly to the hypertensive phenotype. 0

Utilizing a similar strategy, Sabrane, Kuhn, and coworkers have now used a loxP/Tie2-Cre recombination system to selectively delete the gene for GC-A in mouse vascular endothelium (6). The authors show that the direct vasodilating effects of ANP on isolated arteries and apparently normal renal function were preserved in endothelial cell-specific GC-A knockout mice; however, the animals still exhibited a hypertensive phenotype with expanded vascular volume and cardiac hypertrophy. Importantly, ANP infusion did not cause an acute reduction in plasma volume or an increase in albumin loss from the plasma volume even though it exerted these expected effects on control mice. The simplest interpretation of these results is that deletion of the GC-A receptor in endothelial cells attenuates the action of ANP to increase the permeability of the endothelial barrier to plasma proteins. The result is sustained reduction of permeability of the microvessels to plasma proteins (mainly albumin), which are redistributed from the interstitial space to the vascular space. This leads to a change in plasma protein osmotic pressure, which causes an 
A

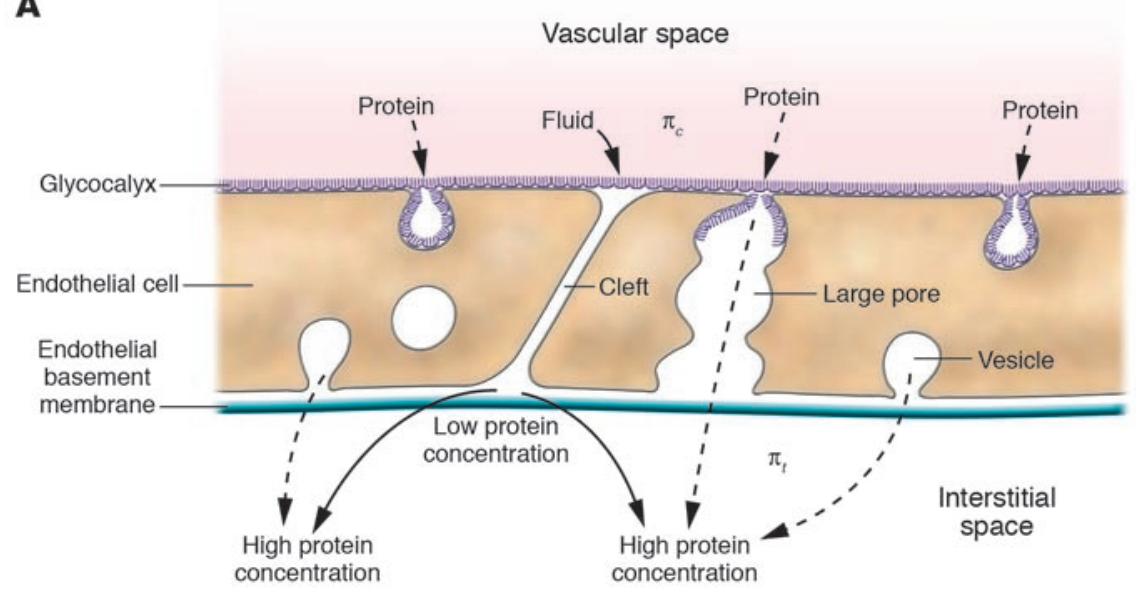

B

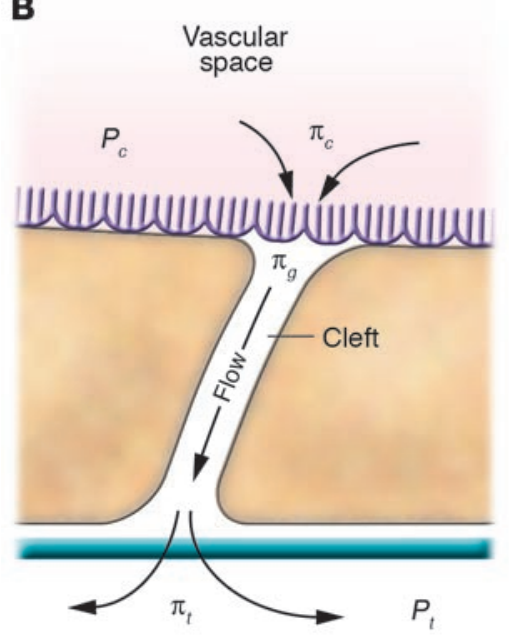

C

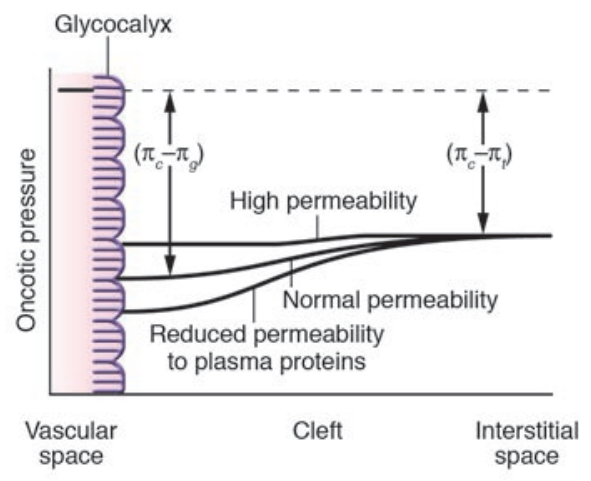

Interstitial space

\section{Figure 1}

Pathways for water and plasma proteins across the endothelial barrier that may be regulated by ANP. (A) Pathways for plasma protein transport across the endothelial permeability barrier that ANP may modulate, including water and plasma transport through the interendothelial cleft at sites where there are infrequent breaks in the junctional strand (see refs. 1,8 ) and specialized vesicular pathways and large pore pathways (1). The plasma protein concentration in the interstitial space is normally about $40 \%$ of that in the plasma. An ANP-induced increase in vascular permeability decreases the difference in plasma protein osmotic pressure between blood and the interstitial space and favors a reduced plasma volume. Conversely, decreased vascular permeability favors increased plasma volume. The latter mechanism would describe results of experiments where the ANP/BNP receptor, GC-A, on vascular endothelial cells is selectively deleted (6). Similar mechanisms are likely to act even more effectively when the endothelial glycocalyx has a low permeability to plasma proteins and contributes to the formation of a plasma ultrafiltrate with low-plasma protein concentration (and low-protein osmotic pressure). (B) Magnified view of the cleft pathway where the plasma protein concentration in the narrow space is lower than the mixed-plasma protein concentration in the interstitial space. Thus, as shown in $\mathbf{C}$, the difference in plasma protein osmotic pressure across the glycocalyx $\left(\pi_{c}-\pi_{g}\right)$ may be significantly larger than the plasma-to-tissue colloid osmotic pressure $\left(\pi_{c}-\pi_{t}\right)$, and an ANP-induced increase in the permeability of the glycocalyx would favor a reduced plasma volume. $\pi_{c}$, plasma protein osmotic pressure; $\pi_{g}$, plasma protein osmotic pressure distal to the glycocalyx; $\pi_{t}$, interstitial space protein osmotic pressure; $P_{c}$, capillary hydrostatic pressure; $P_{t}$, interstitial space hydrostatic pressure.

transport or an increase in the selectivity to plasma protein within individual pathways reduces the average plasma protein concentration in the interstitial space.

It is usually assumed that the osmotic pressure due to the average interstitial protein concentration $\left(\pi_{t}\right)$ can be used in the Starling principle to describe net water flow $\left(J_{v}\right)$.

\section{Equation 1}

$$
J_{v}=\operatorname{LpA}\left[\left(P_{c}-P_{t}\right)-\sigma\left(\pi_{c}-\pi_{t}\right)\right],
$$

ing of protein fluxes and water flows. These differences in flux of water and solute are often ignored, and only an average concentration in the interstitial space considered. Taking this approach as a first approximation, the mechanisms regulating fluid balance in endothelial cell-specific GC-A knockout mice described by Sabrane, Kuhn et al. (6) can be understood if either a reduction in the number of pathways for protein where $P_{c}$ and $P_{t}$ are hydrostatic pressures in the capillaries and interstitial space, respectively, and $\pi_{c}$ is the plasma protein osmotic pressure (also called the colloid osmotic pressure), $L p$ is the hydraulic conductivity of the vessel wall, $A$ is the surface area of the vessel wall, and $\sigma$ is the reflec- 
tion coefficient of the microvascular walls to plasma protein, so that $\sigma\left(\pi_{c}-\pi_{t}\right)$ measures the effective osmotic pressure difference of the plasma proteins between the plasma and the interstitial space. However, it is now understood that the balance of Starling forces involved in the regulation of transvascular fluid balance is far more subtle than previously recognized $(1,8)$. This insight suggests additional ways to interpret the results described in the Sabrane et al. (6) study.

Figure $1 \mathrm{~B}$ illustrates the idea that the difference in plasma osmotic pressure of the plasma proteins involved in transvascular fluid transport is exerted across the endothelial surface glycocalyx, not the whole endothelial barrier as suggested above (see ref. 8 for details). The main pathway for water exchange between the plasma and interstitial space is through the intercellular cleft between adjacent endothelial cells, at sites where there are infrequent narrow breaks in the junctional strands. The glycocalyx on the endothelial cell surface forms an additional series resistance at the entrance to the intercellular cleft on the vascular side. Figure 1B illustrates details of the flow of the plasma ultrafiltrate (low-protein concentration) through the interendothelial cleft in Figure1A at the site of a break in the junctional strand. The glycocalyx has a low but finite permeability to the plasma proteins and is the main permeability barrier to plasma proteins that cross via the endothelial cleft. With this arrangement, the plasma protein concentration, with osmotic pressure $\pi_{g}$ in the very narrow space beneath the glycocalyx can be maintained at a lower level than the protein concentration in the interstitial space (with osmotic pressure $\pi_{t}$ ). Relevant to the results reported by Sabrane et al. (6), the magnitude of $\pi_{g}$ can be modified by increases or decreases in the permeability of the glycocalyx to plasma proteins. It can also be modified by changes in the tissue plasma protein concentration far away from the cleft (because of the back diffusion of the proteins from the interstitial space; see ref. 8 for more details). Thus, as illustrated in Figure $1 \mathrm{C}$, the plasma protein osmotic pressure opposing fluid filtration $\left(\pi_{c}-\pi_{g}\right)$ may be significantly greater than the plasmato-tissue colloid osmotic pressure $\left(\pi_{c}-\pi_{t}\right)$, and it is the magnitude of $\left(\pi_{c}-\pi_{g}\right)$ rather than $\left(\pi_{c}-\pi_{t}\right)$ that should be considered as the principal osmotic pressure determinant of transvascular water flow in the Starling principle. Thus, regulation of the plasma protein permeability of the glycocalyx by ANP-dependent mechanisms may be just as important as other ANP-dependent mechanisms, such as changes in the permeability of vesicular and large-pore pathways illustrated in Figure 1A. This is an area for further investigation.

\section{Future investigations}

The idea that a reduction in vascular permeability to plasma proteins is the primary mechanism explaining the results of Sabrane, Kuhn, and colleagues (6) assumes that there are no significant changes in other variables that might be linked to endothelial functions, including albumin uptake into the endothelial cells controlled by scavenger receptors and microvascular function in the kidney affecting the glomerular filtration of plasma proteins as well as water reabsorption in the vasa recta and peritubular capillaries. As described by Sabrane, Kuhn, and colleagues (6), the loxP/ Tie2-Cre recombination system has been used previously to delete specific genes in endothelium, with this deletion resulting in the loss of such endothelial cell-specific functions as angiogenesis and endothelial cell nitric oxide production. However, the use of this approach to specifically modify vascular permeability is novel and assumes effective deletion of the common ANP/ $\mathrm{BNP}$ receptor in endothelial cells in the microvessels where most water and plasma protein transport occurs (mainly true capillaries and venular capillaries). Although the authors present evidence for deletion of the gene encoding GC-A from vascular endothelium in tissue samples from mouse tail, heart, lung, and aortas and show significantly reduced expression of this receptor in heart and kidney, further studies are needed to measure the effectiveness of the Tie 2 promoter in reducing GC-A expression in microvascular and venular endothelium and in specific fenestrated endothelium such as that associated with the glomerulus and vasa recta.

It is also important to point out that direct measurements of vascular permeability were not made in the experiments described in the present study (6). Changes in accumulation of radiolabeled albumin after 30 minutes of ANP infusion were measured in 9 different tissue types in receptor knockout mice and compared with those in floxed controls. The measured accumulation included albumin in the vascular space, so it is possible that the real levels of interstitial space albumin accumulation were smaller than the total accumulations reported, especially if there was an expanded vascular volume in the microvascular beds of the tissues investigated. Further investigations of real change in permeability and the mechanisms regulating the blood-to-tissue transport of the plasma proteins by ANP in these knockout models will require the use of more refined methods that use 2 tracers for albumin: the first for total accumulation and the second to directly measure the amount of albumin in the vascular spaces in each tissue (see refs. 4, 5).

Further investigators would do well to be guided by the careful evaluations by Sabrane, Kuhn et al. (6) of the renal and cardiac functions in these animals. No significant changes in food, water, and sodium intake or urine, water, or sodium excretion was found in the endothelial cellspecific GC-A knockout animals. If renal mechanisms of salt and water excretion contributed to the observed hypervolumia, then a reduced-salt diet would be expected to attenuate the response. Yet dietary salt restriction had no effect on the hypertensive state. Nevertheless, the study would be strengthened by further investigations of the way in which ANP-independent homeostatic mechanisms (such as renal responses to hypertension and volume expansion) chronically adapt to sustained volume expansion. In addition, reliable measurements of circulating ANP, renin, and aldosterone levels are needed.

In summary, although ANP-dependent modulation of vascular permeability was recognized soon after the discovery of ANP, the importance of changes in vascular permeability in regulating the distribution of water between the plasma space and the interstitial space has not been widely recognized. The elegant study by Sabrane, Kuhn, and colleagues (6) establishes the primary role of ANP-dependent increases in the permeability of the vascular endothelial barrier to plasma proteins in the chronic control of plasma volume. The study also points the way to important new experiments and approaches for investigating in what way defects in ANP-dependent mechanisms regulating endothelial permeability may contribute to the development of cardiovascular disease, including hypertension.

\section{Acknowledgments}

The author thanks Eugene M. Renkin for critical comments and Joyce Lenz for help with the figure. 
Address correspondence to: Fitz-Roy E. Curry, Department of Physiology and Membrane Biology, School of Medicine, One Shields Avenue, University of California Davis, Davis, California 95616, USA. Phone: (530) 752-7081; Fax: (530) 7525423; E-mail: fecurry@ucdavis.edu.

1. Michel, C.C., and Curry, F.E. 1999. Microvascular permeability. Physiol. Rev. 79:703-761.
2. Brenner, B.M., Ballermann, B.J., Gunning, M.E., and Zeidel, M.L. 1990. Diverse biological actions of atrial natriuretic peptide. Physiol. Rev. 70:665-699.

3. Baxter, G.F. 2004. The natriuretic peptides. Basic Res. Cardiol. 99:71-75

4. Tucker, V.L. 1996. Plasma ANP levels and protein extravasation during graded expansion with equilibrated whole blood. Am. J. Physiol. 271:R601-R609.

5. Renkin, E.M., and Tucker, V.L. 1996. Atrial natriuretic peptide as a regulator of transvascular fluid balance. News Physiol. Sci. 11:138-143.

6. Sabrane, K., et al. 2005. Vascular endothelium is critically involved in the hypotensive and hypovolemic actions of atrial natriuretic peptide. J. Clin. Invest. 115:1666-1674. doi:10.1172/JCI23360.

7. Holtwick, R., et al. 2002. Smooth muscle-selective deletion of guanylyl cyclase-A prevents the acute but not chronic effects of ANP on blood pressure. Proc. Natl. Acad. Sci. U. S. A. 99:7142-7147.

8. Adamson R.H., et al. 2004. Oncotic pressures opposing filtration across non-fenestrated rat microvessels. J. Physiol. 557:889-907.

\title{
Lessons learned from cancer may help in the treatment of pulmonary hypertension
}

\section{Serge Adnot}

INSERM U651 and Département de Physiologie, Hôpital H. Mondor, Assistance Publique des Hôpitaux de Paris, Créteil, France.

\begin{abstract}
Hyperplasia of pulmonary artery SMCs (PASMCs) is a pathological hallmark of pulmonary arterial hypertension (PAH). In this issue of the JCI, McMurtry et al. report that adenovirus-mediated overexpression of survivin - a multipotent inhibitor of apoptosis - induces PAH in rats, whereas inhalation of an adenovirus vector encoding a mutant survivin gene with dominantnegative properties reverses established monocrotaline-induced PAH (see the related article beginning on page 1479). These findings raise important issues regarding the role of survivin in the pathogenesis of $\mathrm{PAH}$, its value as a prognostic indicator, and its use as a target for new therapeutic strategies.
\end{abstract}

\section{Pulmonary arterial hypertension and smooth muscle hyperplasia: unresolved questions}

Pulmonary arterial hypertension (PAH), whether idiopathic (iPAH) or associated with underlying diseases (aPAH), is an unexplained condition for which severe forms in adults or neonates are fatal and no satisfactory treatment is available. Valuable new insights into the disease have stemmed in recent years from genetic studies identifying mutations in the gene encoding bone morphogenic protein receptor type 2 (BMPRII) in patients with familial iPAH $(1,2)$ and from functional studies showing dysregulation of molecular events governing pulmonary artery SMC (PASMC) proliferation (3). The nature of the primary defect responsible for triggering, maintaining, and arresting PASMC

Nonstandard abbreviations used: aPAH, PAH associated with underlying diseases; BMP, bone morphogenic protein; BMPRII, bone morphogenic protein receptor type 2; ET-1, endothelin-1; 5-HHT, serotonin transporter; 5-HT, serotonin; iPAH, idiopathic PAH; PAH, pulmonary arterial hypertension; PASMC, pulmonary artery SMC.

Conflict of interest: The author has declared that no conflict of interest exists.

Citation for this article: J. Clin. Invest. 115:1461-1463 (2005). doi:10.1172/JCI25399. proliferation in PAH is poorly understood but may be either an inherent characteristic of PASMCs (as seen with cancer cells) or a secondary response to an external abnormality, such as upregulation of a growth factor. Under the first hypothesis, cells removed from their in vivo environment would continue to proliferate excessively, whereas under the second hypothesis, their proliferation rate would be normal. An intermediate situation has been found: when removed from their in vivo environment, PASMCs from PAH patients demonstrate excessive proliferation when stimulated by serotonin (5-hydroxytryptamine [5-HT]) or endothelin-1 (ET-1) but not when stimulated by PDGF, EGF, FGF, IGF, or TGF- $\beta(4,5)$. Moreover, they are far less sensitive to growth inhibition by bone morphogenic proteins (BMPs) than are normal cells (6). Therefore, the intrinsic abnormalities of these PASMCs involve not intracellular mitogenic signals but rather signal transmission from specific autocrine or paracrine factors such as BMPs, 5-HT, or ET-1. Increased expression of the serotonin transporter (5-HTT), which mediates the proliferative effect of 5-HT, and marked reduction of BMPRII expression appear specific to $\mathrm{PAH}$ and
aPAH $(4,7)$. These molecular alterations occur without apparent genetic abnormalities: in cells without the mutation in BMPRII, the extent of BMPRII underexpression is similar to that in mutated cells (6), and the 5-HTT overexpression can be explained only partly by the polymorphism of its gene promoter (5). Why PASMCs from $\mathrm{PAH}$ patients maintain an abnormal phenotype in vitro and how environmental and genetic factors regulate the expression of these key molecular transmitters remain open questions.

Until now, alterations in apoptotic processes have not been considered to play a role in PASMC proliferation, in contrast to mechanisms of disorganized EC proliferation contributing to plexiform lesions at branch points of pulmonary arteries in iPAH (8). In this issue of the JCI, McMurtry et al. open up the intriguing possibility that increased survivin expression may contribute to the abnormal PASMC phenotype observed in PAH (9).

\section{Is there a role for survivin} in the pathogenesis of PAH?

Survivin belongs to the inhibitor of apoptosis protein (IAP) family, members of which confer protection from deathinducing stimuli (10). One of the most striking features of survivin is its differential expression in cancerous versus normal tissues. Survivin is virtually undetectable in normal adult differentiated tissues but is expressed in most human cancers (11). Thus, dysregulated survivin expression is considered to be a major pathologic mechanism of apoptosis inhibition. However, survivin pathway reactivation may occur 